\title{
Clinical outcomes of residual or recurrent nasopharyngeal carcinoma treated with endoscopic nasopharyngectomy plus chemoradiotherapy or with chemoradiotherapy alone: a retrospective study
}

Jingjin Weng ${ }^{1,2}$, Jiazhang Wei ${ }^{2}$, Jinyuan Si ${ }^{2}$, Yangda Qin ${ }^{2}$, Min Li ${ }^{2}$, Fei Liu ${ }^{3}$, Yongfeng Si Corresp., ${ }^{2}$, Jiping Su Corresp. 1

${ }^{1}$ Department of Otolaryngology-Head and Neck Surgery, First Affiliated Hospital of Guangxi Medical University, Nanning, China

2 Department of Otolaryngology \& Head and Neck Oncology, The People's Hospital of Guangxi Zhuang Autonomous Region, Nanning, China

3 Research Center of Medical Sciences, The People's Hospital of Guangxi Zhuang Autonomous Region, Nanning, China

Corresponding Authors: Yongfeng Si, Jiping Su

Email address: syfklxf@126.com, ymsu2@126.com

Background. Local residual and recurrent nasopharyngeal carcinoma (NPC) generally shows treatment failure after standard radiotherapy with or without concurrent chemotherapy. Whether endoscopic nasopharyngectomy might provide an additional therapeutic advantage remains controversial. Therefore, we retrospectively compared the clinical prognoses of patients with residual or recurrent NPC treated with endoscopic nasopharyngectomy combined with chemoradiotherapy (CRT) with those of patients treated with CRT alone. Methods and Materials. A total of sixty-two patients with local residual or recurrent NPC were studied retrospectively: 36 patients received endoscopic nasopharyngectomy combined with CRT, whereas 26 patients who refused the surgery or had surgical contraindications received CRT alone. Serum Epstein-Barr virus (EBV) DNA levels were measured pre- and post-treatment. The differences in prognosis between the two treatment regimens and the pre- and post-treatment changes in EBV-DNA levels were analyzed. Results. The median follow-up time was 31 months, with a 3-year overall survival (OS) of $51.40 \%$ and a 3-year disease-free survival (DFS) of $46.86 \%$. The surgery+ CRT group had a better OS than the CRT alone group did $\left(\chi^{2}=4.054, P=0.044\right)$. The pretreatment EBV-DNA levels showed a positive correlation with the clinical staging of recurrent NPC $\left(\chi^{2}=11.674, P=0.009\right)$. Patients with negative pretreatment serum EBV-DNA levels showed a superior OS to those of patients who tested positive for EBV-DNA ( $>0$ copy $/ \mathrm{mL})\left(\chi^{2}=9.833, P=0.002\right)$. The post-treatment EBV-DNA levels, compared with the pretreatment levels, decreased significantly in the surgery + CRT group ( $Z=-3.484$, $P=0.000$ ). In contrast, the EBV-DNA levels after CRT alone did not decrease significantly $(Z=-1.956, P=0.051)$. Multivariate analysis indicated that local staging, pretreatment EBVDNA load, and the treatment method were independent risk factors for OS. Subgroup 
analysis indicated that the patients who tested negative for EBV-DNA before the treatment and those who received surgery + CRT showed a better OS than those who received CRT alone. Conclusions. The pretreatment serum EBV-DNA level was associated with disease prognosis. The combination therapy preceded by surgery can effectively decrease the copy number of EBV-DNA. Patients with local intermediate- and late-stage NPC, especially those negative for EBV-DNA, may consider opting for surgery followed by post-operative adjuvant radiotherapy or chemotherapy. 


\section{Clinical outcomes of residual or recurrent nasopharyngeal carcinoma treated}

2 with endoscopic nasopharyngectomy plus chemoradiotherapy or with

3 chemoradiotherapy alone: a retrospective study

5 Jingjin Weng ${ }^{1,2}$, Jiazhang Wei ${ }^{2}$, Jinyuan $\mathrm{Si}^{2}$, Yangda $\mathrm{Qin}^{2}$, Min $\mathrm{Li}^{2}$, Fei Liu ${ }^{3}$, Yongfeng $\mathrm{Si}^{2 \#}$,

6 Jiping $\mathrm{Su}^{1 \#}$

$7 \quad{ }^{1}$ Department of Otolaryngology-Head and Neck Surgery, First Affiliated Hospital of Guangxi

8 Medical University, Nanning, China

$9 \quad$ 2Department of Otolaryngology \& Head and Neck Oncology, The People's Hospital of Guangxi

10 Zhuang Autonomous Region, Nanning, China

$11{ }^{3}$ Research Center of Medical Sciences, The People's Hospital of Guangxi Zhuang Autonomous

12 Region, Nanning, China

$14{ }^{\#}$ Corresponding authors:

15 Yongfeng Si, Department of Otolaryngology \& Head and Neck Oncology, The People's Hospital

16 of Guangxi Zhuang Autonomous Region, No. 6 Taoyuan Road, Nanning 530021, People's

17 Republic of China. Tel.: +86-771-2186318, Email:syfklxf@126.com

19 Jiping Su, Department of Otolaryngology-Head and Neck Surgery, First Affiliated Hospital of

20 Guangxi Medical University, No. 6 Shuangyong Road, Nanning 530021, People’s Republic of

21 China, Tel.: +86-771-5356511, Email: ymsu2@126.com 
23 Abstract

Background. Local residual and recurrent nasopharyngeal carcinoma (NPC) generally shows treatment failure after standard radiotherapy with or without concurrent chemotherapy. Whether endoscopic nasopharyngectomy might provide an additional therapeutic advantage remains controversial. Therefore, we retrospectively compared the clinical prognoses of patients with residual or recurrent NPC treated with endoscopic nasopharyngectomy combined with chemoradiotherapy (CRT) with those of patients treated with CRT alone.

Methods and Materials. A total of sixty-two patients with local residual or recurrent NPC were studied retrospectively: 36 patients received endoscopic nasopharyngectomy combined with CRT, whereas 26 patients who refused the surgery or had surgical contraindications received CRT alone. Serum Epstein-Barr virus (EBV) DNA levels were measured pre- and post-treatment.

The differences in prognosis between the two treatment regimens and the pre- and post-treatment changes in EBV-DNA levels were analyzed.

Results. The median follow-up time was 31 months, with a 3 -year overall survival (OS) of 51.40\% and a 3-year disease-free survival (DFS) of $46.86 \%$. The surgery+ CRT group had a better OS than the CRT alone group did $\left(\chi^{2}=4.054, \mathrm{P}=0.044\right)$. The pretreatment EBV-DNA levels showed a positive correlation with the clinical staging of recurrent NPC $\left(\chi^{2}=11.674, \mathrm{P}=0.009\right)$. Patients with negative pretreatment serum EBV-DNA levels showed a superior OS to those of patients

41 who tested positive for EBV-DNA $(>0$ copy $/ \mathrm{mL})\left(\chi^{2}=9.833, \mathrm{P}=0.002\right)$. The post-treatment EBV- 
43 CRT group $(Z=-3.484, P=0.000)$. In contrast, the EBV-DNA levels after CRT alone did not

44 decrease significantly $(Z=-1.956, P=0.051)$. Multivariate analysis indicated that local staging,

45 pretreatment EBV-DNA load, and the treatment method were independent risk factors for OS.

46 Subgroup analysis indicated that the patients who tested negative for EBV-DNA before the

47 treatment and those who received surgery + CRT showed a better OS than those who received

48 CRT alone.

49 Conclusions. The pretreatment serum EBV-DNA level was associated with disease prognosis.

50 The combination therapy preceded by surgery can effectively decrease the copy number of EBV-

51 DNA. Patients with local intermediate- and late-stage NPC, especially those negative for EBV-

52 DNA, may consider opting for surgery followed by post-operative adjuvant radiotherapy or

53 chemotherapy. 


\section{Introduction}

Nasopharyngeal carcinoma (NPC) is a common malignant disease in the southern regions of

China. Because NPC is sensitive to irradiation during the initial treatment, radiotherapy is the

preferred treatment. However, approximately $10 \%$ of patients show local recurrence after

radiotherapy(Yu et al. 2005). After the first course of radiotherapy, patients often present with

fibrous hyperplasia of the nasopharynx and poor local circulation due to local vascular occlusion.

This condition can lead to low efficacy of the treatment and many side effects from re-irradiation

alone. In addition, the residual tumor after the first treatment may acquire mutations or some

other mechanisms that are likely to persist and render the tumor resistant to the same treatment.

Therefore, surgical treatment of residual and recurrent NPC has been advocated(Chan \& Wei 2012).

tumors, endoscopic nasopharyngectomy is less invasive and does not result in facial scars.

Therefore, a number of institutions have begun performing endoscopic nasopharyngectomy for

the resection of residual and recurrent $\mathrm{NPC}(\mathrm{Chen}$ et al. 2009; Zou et al. 2015). However, a

previous study has shown that the serum level of Epstein-Barr virus DNA (EBV-DNA) is an

effective indicator for monitoring the therapeutic efficacy in patients with recurrent NPC(An et

al. 2011). Nonetheless, there is still a group of patients with residual or recurrent NPC in whom 
EBV-DNA cannot be detected. The suitability of surgery for these patients and whether surgery can be performed in late-stage recurrent NPC have rarely been reported in the literature. Thus, this retrospective study was carried out to determine the plasma levels of EBV-DNA in patients with residual or recurrent NPC to explore any variations and their association with the disease prognosis.

\section{Materials and Methods}

\section{Study criteria and patient characteristics}

Data for the patients with residual or recurrent NPC were retrieved from the database of The People's Hospital of Guangxi Zhuang Autonomous Region (Nanning, China). All the patients recruited in this study provided written informed authorization consent, in which they agreed to use of their clinical and imaging data for non-commercial scientific research. We adhered to the bioethics principles of the Declaration of Helsinki. This retrospective study was approved by the ethics committee of The People's Hospital of Guangxi Zhuang Autonomous Region (Ethical Application Ref: Keyan-Guangxi-Keji-2016-31).

The case inclusion criteria included (1) patients admitted to The People's Hospital of Guangxi Zhuang Autonomous Region with residual or recurrent NPC. The diagnoses of residual or recurrent NPC for every case included in our study were strictly confirmed by pathological examination. The staging for each patient was based upon the $\mathrm{T}$ classification of residual or recurrent tumors. A residual tumor was defined as a persistent lesion in the nasopharynx within 6 months after full-dose radiotherapy. A recurrent tumor was defined as a local recurrence after 
106

107

complete remission within 6 months after full-dose radiotherapy and the subsequent emergence of new lesions in the nasopharynx after 6 months. Additional inclusion criteria were (2) a measurable lesion with evaluable efficacy, (3) a Karnofsky score $\geq 70$, and (4) provision of written informed consent to undergo surgery and/or chemoradiotherapy. The case exclusion criteria were (1) patients with cervical lymph node metastasis or distant metastasis, (2) patients with severe damage to liver and kidney function, and (3) patients with severe immune deficiency.

3

A total of 62 patients with residual or recurrent NPC who were treated at our hospital between June 2011 and April 2013 were enrolled in the study (5 cases of residual tumors and 57 cases of recurrent tumors). The patients included 47 men and 15 women, aged 22 to 70 years with a median age of 50 years. Of these 62 patients, 58 patients were pathologically identified as having undifferentiated non-keratinizing carcinoma (WHO type III), three patients had keratinizing squamous cell carcinoma (WHO type I), and only one patient had differentiated non-keratinizing carcinoma (WHO type II).

In general, surgery was recommended and preferred for all the regional residual or recurrent cases, except when the following exclusion criteria were encountered: (1) internal carotid artery encasement, massive intracranial intradural involvement, or orbital space invasion; (2) uncontrolled nasopharyngeal skull infection; (3) general anesthesia surgery contraindications; or (4) patient refusal to undergo surgery. To retrospectively analyze the clinical outcome of the patients who underwent endoscopic nasopharyngectomy, the patients were divided into two groups according to the treatment selection. In the surgical group, 36 patients underwent 
127

128

129

130

131

132

133

134

135

136

137

138

139

140

141

142

143

144

145

146

147

complete endoscopic nasopharyngectomy of the residual or recurrent tumor and subsequent postoperative chemoradiotherapy (CRT). In the CRT alone group, 26 patients received concurrent radiotherapy and chemotherapy. The patients' sex, age, local staging, and EBV-DNA positive rate showed no statistically significant differences between the two groups (all P>0.05) (Table 1).

\section{Treatment methods}

The surgical group received endoscopic nasopharyngectomy plus concurrent radiotherapy and a chemotherapy treatment regimen. Surgery was performed under general anesthesia. The patients were placed in a supine position, and a cotton swab soaked with epinephrine was used to shrink the nasal mucosa 2 - 3 times. Under the guidance of nasal endoscopy, an electric knife was then used to resect the nasopharyngeal tumor via a nasal approach. The resection margin was defined according to the results of nasopharyngeal skull-base magnetic resonance imaging (MRI) and intraoperative observations. Typical imaging data of the patients are shown in Figure 1. According to post-operative pathological examinations, all the resections were confirmed to be residual or recurrent tumors and three patients in the surgery group $(8.3 \%, 3 / 36)$ were found to have positive margins.

The chemotherapy regimen and doses for the surgical + CRT and CRT alone group were the same and consisted of $30 \mathrm{mg} / \mathrm{m}^{2}$ cisplatin via intravenous infusion beginning on days $1-3$ and $1000 \mathrm{mg} / \mathrm{m}^{2}$ gemcitabine via intravenous infusion on day 1 and day 8; each cycle lasted 21days, and 2-4 cycles were administered consecutively. For the intensity-modulated radiotherapy 
148 (IMRT) for both groups, the target area was delineated on the basis of the tumor boundary shown

149 in MRI, where in the gross tumor volume (GTV) was irradiated with 2.0Gy/fraction 5days/week;

150 the total dose of GTV was 60-66Gy. The median radiotherapy dose for primary NPC was 71.2

151 (52-81) Gy. The median time span between the primary treatment and treatment of the

152 recurrence was $20(3-178)$ months.

153

154

155

156

157

158

159

160

161

162

163

164

165

166

167

168

\section{Collection of peripheral blood samples and EBV-DNA load assay}

A $2 \mathrm{~mL}$ blood sample was collected from each patient's antecubital vein pre- and posttreatment in an anticoagulation tube containing EDTA, centrifuged to separate the blood plasma into the upper phase for DNA extraction, and stored at $-20^{\circ} \mathrm{C}$ for later use. A Light-Cycler 480 quantitative fluorescence PCR platform (Roche Diagnostics, Basel, Switzerland) was used to quantify EBV-DNA levels. The test kit was provided by the Da'an Gene Diagnostic Center of Sun Yat-Sen University (Cat. \# DA-D065). The target gene for amplification was derived from the BamHI-W fragment of EBV. All procedures were performed according to the manufacturer's protocol. EBV-DNA $>0$ copies/mL was considered EBV-positive. EBV-DNA(+) refers to the pretreatment EBV-DNA-positive level unless otherwise stated.

\section{Follow-up and outcome measures}

The patients began regular follow-up examinations after the treatment ended. During the first year after treatment, the patients returned to the hospital once every 3 months for examination as inpatients or outpatients. After 1 year, they received follow-up examinations once every 6 months, and after 3 years, they received follow-up examinations once every 12 months. The date of the final follow-up visit was 30 July 2016; the median follow-up time was 31 months, 
169 and the follow-up rate was $100 \%$.

170 Statistical analysis

171 The SPSS 18.0 statistical software package (SPSS Inc., Chicago, IL, USA) was used to

172 establish the database. Between-group comparisons of the count data were performed using the

$173 \chi^{2}$ test. Pre- and post-treatment comparisons of EBV-DNA levels were performed using the

174 Wilcoxon rank-sum test. Comparisons of EBV-DNA levels among different T-stages were

175 performed using the Kruskal-Wallis H test. Kaplan-Meier survival curves were used for

176 univariate survival analysis, and the log-rank test was performed for the between-group comparison of survival curves. Multivariate analysis was performed using Cox regression analysis. $\mathrm{P}<0.05$ indicated that the differences were statistically significant.

\section{Results}

Association of EBV-DNA with the clinical stages and changes in EBV-DNA pre- and post-

\section{treatment}

The EBV-DNA positive rate in the serum of all patients was $43.55 \%$. The mean EBV-DNA copy numbers of patients with stages rT1, rT2, rT3, and rT4 disease were 510, 583, 956, and 3909 copies/mL, respectively. The EBV-DNA levels increased with NPC stages $\left(\chi^{2}\right.$ value $=11.674, \mathrm{P}=0.009$ ) (Figure $2 \mathrm{~A}$ ). The EBV-DNA levels decreased significantly in the surgery + post-operative CRT group $(\mathrm{Z}=-3.484, \mathrm{P}<0.001)$; compared with the pretreatment levels, the post-treatment EBV-DNA levels in the CRT group did not decrease significantly $(Z=-1.956$,

$\mathrm{P}=0.051)$ (Figure 2B). 


\section{Prognosis analysis}

The 3-year overall survival (OS) for all patients was $51.40 \%$, and the 3-year disease-free survival (DFS) was $46.86 \%$. The univariate analysis indicated that local staging, treatment method, and pre- and post-treatment EBV-DNA levels correlated with the OS and DFS (all $\mathrm{P}<0.05$ ) (Table 2). Kaplan-Meier survival analysis indicated that the prognosis of patients with local early-stage NPC (T1+T2) was significantly better than that of patients with local late-stage NPC (T3+T4) (Figure 3A and 4A). The prognosis of the surgery + CRT group was superior to that of the CRT alone group (Figure 3B and 4B). Pre- and post-treatment EBV-DNA levels in the peripheral blood were associated with the clinical prognosis (Figure 3C, 3D, 4C and 4D). Notably, the patients with post-treatment EBV-positive disease were found to had obviously worse outcomes (Figure 3D and 4D), which indicated that post-treatment EBV-positive strongly suggests a poor prognosis in patients with residual or recurrent NPC. Multivariate analysis indicated that local staging, pretreatment EBV-DNA levels, and the treatment method were independent risk factors for OS, whereas local staging and the treatment method were independent risk factors for DFS (Table 3). By the end of our follow-up, a total of 29 patients had died, of whom17 died of local recurrence, 7 died of distant metastasis, 3 died of internal carotid artery rupture caused by skull necrosis, and 2 died of cervical recurrence.

\section{Subgroup analysis}

The patients were divided into subgroups on the basis of local staging and the treatment method. The 4 subgroups were the local early-stage (T1+T2) and surgery + CRT subgroup (17 cases), the local early-stage (T1+T2) and CRT alone subgroup (12 cases), the local late-stage 
211 (T3+T4) and surgery + CRT subgroup (19 cases), and the local late-stage (T3+T4) and CRT

212 alone subgroup (14 cases). The results indicated that for the local early-stage subgroups, the OS

213 and DFS did not show significant differences among the different treatment methods (all $\mathrm{P}>0.05)$

214 (Figure 5A and 5B). For the local late-stage subgroups, surgery + CRT showed a better DFS than

215 CRT alone $(\mathrm{P}=0.032)$, whereas $\mathrm{OS}$ did not show a significant difference $(\mathrm{P}>0.05)$ (Figure $5 \mathrm{C}$ and 216 5D).

217 The patients were also divided into subgroups on the basis of the presence of EBV-DNA 218 and the treatment method. The 4 subgroups were the EBV-DNA negative and surgery + CRT 219 subgroup (19 cases), the EBV-DNA negative and CRT alone subgroup (16 cases), the EBV220 DNA positive and surgery + CRT subgroup (17 cases), and the EBV-DNA positive and CRT 221 alone subgroup (10 cases). The results indicated that the EBV-DNA negative and surgery + CRT 222 subgroup had better OS and DFS than the CRT alone subgroup (OS:P=0.009, DFS:P=0.003) 223 (Figure 6A and 6B). Among the patients who tested positive for EBV-DNA, the OS and DFS did 224 not show significant differences between the 2 treatment regimens (all $\mathrm{P}>0.05$ ) (Figure $6 \mathrm{C}$ and $2256 \mathrm{D})$.

\section{Complications from surgery and chemoradiotherapy}

In the surgical group, the most common complications from endoscopic surgery and/or radiotherapy were secretory otitis media $(47.2 \%, 17 / 36)$, temporal lobe necrosis $(25.0 \%, 9 / 36)$,

229 skull base necrosis $(19.4 \%, 7 / 36)$, cranial nerve palsy $(13.9 \%, 5 / 36)$ and nasopharyngeal 230 hemorrhage $(5.6 \%, 2 / 36)$. In the non-surgical (CRT alone) group, the common complications 231 from radiotherapy were temporal lobe necrosis $(53.9 \%, 14 / 26)$, secretory otitis media $(46.2 \%$, 
232 12/26), cranial nerve palsy $(23.1 \%, 6 / 26)$, and skull base necrosis $(15.4 \%, 4 / 26)$. The most 233 common side-effects from chemotherapy in the both group were hematological toxicities, 234 including leukopenia, neutropenia, and thrombocytopenia. Other non-hematological toxicities 235 included mild transaminase elevation and tolerable nausea and vomiting. In general, the chemotherapy-related side effects were tolerable and could be well-controlled.

\section{Discussion}

NPC is a common malignant tumor found in the Guangdong and Guangxi regions of China.

According to the World Health Organization (WHO) pathological classification of tumors, NPC

can be divided into WHO types I, II, and III. The most common pathological type is undifferentiated, non-keratinizing carcinoma (WHO type III), which is also associated with EBV infection. The EBV-DNA levels are related to the stage, volume, and prognosis of NPC at the initial treatment. Previous studies have shown that EBV-DNA levels are not detectable after treatment in patients with a first diagnosis of NPC, whereas detectable levels of EBV-DNA in the blood often indicate the presence of residual or recurrent NPC(Lin et al. 2004). In our patient group, the detection rate of EBV-DNA was $43.55 \%$. With regard to the association between

EBV-DNA levels and the stages of recurrent NPC, a study has found that EBV-DNA levels are related to tumor staging(Chan \& Wong 2014). Furthermore, on the basis of our data, the average

EBV-DNA load for stage rT4 was 3,908 copies/mL, whereas that at stage rT1 was only 510 copies/mL. These results indicated that EBV-DNA may facilitate the assessment of clinical stages, in agreement with findings from a previous report(Chang et al. 2012). Further analysis 
253 254 255 256 257

revealed that the prognosis of patients who tested negative for EBV-DNA was superior to that of the EBV-DNA-positive group. Thus, when clinically evaluating a patient's condition, we recommend assessment of patient EBV-DNA levels in addition to clinical staging. A recent study has shown that EBV-DNA is an independent risk factor for the prognosis of NPC at the time of initial treatment(Peng et al. 2016). The recent National Comprehensive Cancer Network(NCCN) guidelines have also recommended including EBV-DNA in the staging of NPC at the initial treatment. According to our present results, we agree that EBV-DNA should also be included in the staging of recurrent NPC.

A subsequent analysis has shown that patients with residual or recurrent NPC who test negative for EBV-DNA show better responses after surgery, whereas patients who test positive for EBV-DNA show similar responses after surgical treatment and CRT. However, patients receiving repeat radiotherapy have a poorer quality of life than those who undergo surgery(You et al. 2015). A number of studies have supported the claim that patients who test negative for EBV-DNA have better prognoses(Shen et al. 2015; Stoker et al. 2016), and have advocated surgery as the main treatment method(You et al. 2015). Unlike other studies in which the pretreatment EBV-DNA levels of recurrent NPC have been found to be predominantly positive(Chan et al. 2012), only 27 patients tested positive for EBV-DNA in our patient group. Therefore, the finding that the prognosis of the EBV-DNA-positive group was not better in the surgical group than in the non-surgical group may have been because of the small number of cases analyzed. 
273 Unlike NPC at the initial diagnosis, the preferred treatment for recurrent NPC is often 274 surgery, because it provides a better prognosis than CRT(You et al. 2015). Furthermore, in terms 275 of resectable lesions, surgery is essentially able to achieve complete resection of the observable tumor. This study found that the EBV-DNA levels of the surgical group had decreased significantly, whereas those of the CRT alone group did not decrease significantly. Some studies have shown that blood EBV originates from tumor cells(Lin et al. 2004; Wang et al. 2010). It is known that plasma EBV levels are proportional to the viral production and viral release from the tumor cells and inversely proportional to the clearance rate of blood circulation. Therefore, the surgical resection of the tumor would lead to a significant reduction in EBV, thus causing a rapid decline in EBV-DNA levels. Through the continuous monitoring of post-operative EBV levels, researchers have found that the median clearance time is $139 \mathrm{~min}$ (To et al. 2003). Data from follow-up visits have revealed that patients with high pretreatment EBV-DNA levels have poorer prognoses(An et al. 2011). Furthermore, the continuous monitoring of EBV-DNA levels has shown that its clearance rate is related to the prognosis of recurrent NPC(Wang et al. 2010). In this study, EBV-DNA levels showed no significant differences between the surgery group and the CRT group before treatment; however, they decreased significantly after surgical treatment. This finding further suggests that surgical treatment was more effective than CRT at facilitating the removal of EBV, thus decreasing the probability of reactivating EBV. This EBV removal may be one of the reasons why surgical treatment had better therapeutic efficacy than CRT alone. However, surgery is not feasible in every case of recurrent nasopharyngeal carcinoma. For instance, some patients have contraindications for endoscopic nasopharyngectomy, such as 
294

295

296

297

298

299

300

301

302

303

304

305

306

307

308

309

310

311

312

313

314

internal carotid artery encasement, massive intracranial intradural involvement, and orbital

content invasion(Castelnuovo et al. 2013). Thus, only the carefully chosen patients would benefit

from surgery.

Staging is an independent risk factor for patients with recurrent NPC(Hua et al. 2012). We

obtained similar results in our patient group, which showed that early-stage patients had better

prognoses than late-stage patients. For early-stage patients, the surgical treatment had definite

therapeutic efficacy, whereas CRT also achieved a satisfactory effect. For late-stage patients, the

effective dose at the tumor center is relatively low, and radiotherapy often leads to residual tumor.

Our data showed that among patients with local late-stage NPC, those who underwent surgical

treatment had superior DFS, as compared with those who received CRT only $(\mathrm{P}=0.032)$. This

finding indicates that surgical treatment is still suitable for patients with local late-stage NPC and

that it can achieve better therapeutic efficacy than non-surgical treatment. There were no

significant differences in OS between the 2 groups, possibly because the patient deaths occurred

because of other complications such as massive epitasis. Most previous studies have reported

that surgery should be performed only in early-stage recurrent NPC. However, a recent study has

shown that surgery has good efficacy for local intermediate-stage NPC(Wong et al. 2017).

Currently, the indications for surgical treatment and CRT in local recurrent NPC have not

yet been clearly defined. In contrast, surgery is advocated as the main treatment method for the

recurrence of lymph node metastases, because they can essentially be completely resected

surgically and rarely leads to severe complications. However, complete surgical resection may

not be achieved in NPC because of the complex anatomical location of the nasopharynx, the 
315 presence of important nerves and vessels in the vicinity, and the wide area of invasion in local

316 late-stage recurrence. Therefore, previous studies have advocated that surgical treatment should 317 mainly be reserved for rT1-T2 patients(You et al. 2015). With regard to local early-stage NPC, a

318 previous study has reported that CRT can achieve satisfactory efficacy(Qiu et al. 2012). That

319 study has also shown that the therapeutic efficacies of surgery and CRT are similar in early-stage

patients. However, there is a lack of randomized control trials comparing surgical and nonsurgical treatments and a paucity of relevant meta-analyses.

Our study was a single-center and small-cohort study, the findings presented in our study should be viewed as exploratory and needs to be further confirmed in subsequent studies. Our study was also a retrospective study in which the group allocation was based on the patient treatment wishes and with surgical contraindications or not, a design that may have led to bias.

For ethical considerations, it may be difficult to perform a completely randomized controlled clinical trial to evaluate the additional benefits in the patients with residual or recurrent NPC undergoing endoscopic nasopharyngectomy combined with chemoradiotherapy compared with those receiving chemoradiotherapy alone. Even so, a prospective long-term follow-up and wellbalanced cohort studies involving a larger sample number from multicenter should be conducted to more carefully evaluate the superiority of endoscopic surgery in addition to CRT in patients with residual or recurrent NPC.

\section{Conclusions}

335 On the basis of the findings presented in our study, we suggest that serum EBV-DNA load is 
336

337

338

339

340

341

\section{2}

343

344

345

346

347

348

349

350

351

352

353

354

355

356

357

358

359

360

361

362

363

364

365

related to the stage of recurrent NPC. The combination therapy preceded by surgery can effectively decrease the copy number of EBV-DNA, and its efficacy is superior to that of conventional CRT alone. Patients with local intermediate and late-stage NPC, especially those who test negative for EBV-DNA, may consider opting for surgical treatment followed by postoperative adjuvant radiotherapy or chemotherapy.

\section{Acknowledgements}

We are thankful for the participations of every enrolled patient in this study.

\section{References}

An X, Wang FH, Ding PR, Deng L, Jiang WQ, Zhang L, Shao JY, and Li YH. 2011. Plasma Epstein-Barr virus DNA level strongly predicts survival in metastatic/recurrent nasopharyngeal carcinoma treated with palliative chemotherapy. Cancer 117:3750-3757. 10.1002/cncr.25932

Castelnuovo P, Nicolai P, Turri-Zanoni M, Battaglia P, Bolzoni Villaret A, Gallo S, Bignami M, and Dallan I. 2013. Endoscopic endonasal nasopharyngectomy in selected cancers. Otolaryngol Head Neck Surg 149:424-430. $10.1177 / 0194599813493073$

Chan JY, Chow VL, Mok VW, Ho AC, and Wei WI. 2012. Prediction of surgical outcome using plasma EpsteinBarr virus dna and (18)F-FDG PET-CT scan in recurrent nasopharyngeal carcinoma. Head Neck 34:541545. 10.1002/hed.21768

Chan JY, and Wei WI. 2012. Critical appraisal of maxillary swing approach for nasopharyngeal carcinoma. Expert Opin Ther Targets 16 Suppl 1:S111-117. 10.1517/14728222.2011.637920

Chan JY, and Wong ST. 2014. The role of plasma Epstein-Barr virus DNA in the management of recurrent nasopharyngeal carcinoma. Laryngoscope 124:126-130. 10.1002/lary.24193

Chang KP, Tsang NM, Liao CT, Hsu CL, Chung MJ, Lo CW, Chan SC, Ng SH, Wang HM, and Yen TC. 2012. Prognostic significance of 18F-FDG PET parameters and plasma Epstein-Barr virus DNA load in patients with nasopharyngeal carcinoma. J Nucl Med 53:21-28. 10.2967/jnumed.111.090696

Chen MY, Wen WP, Guo X, Yang AK, Qian CN, Hua YJ, Wan XB, Guo ZM, Li TY, and Hong MH. 2009. Endoscopic nasopharyngectomy for locally recurrent nasopharyngeal carcinoma. Laryngoscope 119:516522. 10.1002/lary. 20133 
Hua YJ, Han F, Lu LX, Mai HQ, Guo X, Hong MH, Lu TX, and Zhao C. 2012. Long-term treatment outcome of recurrent nasopharyngeal carcinoma treated with salvage intensity modulated radiotherapy. Eur $J$ Cancer 48:3422-3428. 10.1016/j.ejca.2012.06.016

Lin JC, Wang WY, Chen KY, Wei YH, Liang WM, Jan JS, and Jiang RS. 2004. Quantification of plasma EpsteinBarr virus DNA in patients with advanced nasopharyngeal carcinoma. $N$ Engl $J$ Med 350:2461-2470. 10.1056/NEJMoa032260

Peng H, Guo R, Chen L, Zhang Y, Li WF, Mao YP, Sun Y, Zhang F, Liu LZ, Lin AH, and Ma J. 2016. Prognostic Impact of Plasma Epstein-Barr Virus DNA in Patients with Nasopharyngeal Carcinoma Treated using Intensity-Modulated Radiation Therapy. Sci Rep 6:22000. 10.1038/srep22000

Qiu S, Lin S, Tham IW, Pan J, Lu J, and Lu JJ. 2012. Intensity-modulated radiation therapy in the salvage of locally recurrent nasopharyngeal carcinoma. Int $J$ Radiat Oncol Biol Phys 83:676-683. 10.1016/j.ijrobp.2011.07.006

Shen T, Tang LQ, Luo DH, Chen QY, Li PJ, Mai DM, Guo SS, Liu LT, Qian CN, Guo X, Zeng MS, Mo HY, and Mai HQ. 2015. Different prognostic values of plasma Epstein-Barr virus DNA and maximal standardized uptake value of 18F-FDG PET/CT for nasopharyngeal carcinoma patients with recurrence. PLoS One 10:e0122756. 10.1371/journal.pone.0122756

Stoker SD, Wildeman MA, Novalic Z, Fles R, van der Noort V, de Bree R, Braunius WW, van den Broek GB, Kreike B, Kross KW, Juwana H, Ramayanti O, Verkuijlen SA, de Boer JP, Greijer AE, Middeldorp JM, and Tan IB. 2016. Can Epstein-Barr virus DNA load in nasopharyngeal brushings or whole blood predict recurrent nasopharyngeal carcinoma in a non-endemic region? A prospective nationwide study of the Dutch Head and Neck Oncology Cooperative Group. Eur Arch Otorhinolaryngol 273:1557-1567. 10.1007/s00405-015-3620-y

To EW, Chan KC, Leung SF, Chan LY, To KF, Chan AT, Johnson PJ, and Lo YM. 2003. Rapid clearance of plasma Epstein-Barr virus DNA after surgical treatment of nasopharyngeal carcinoma. Clin Cancer Res 9:3254-3259.

Wang WY, Twu CW, Chen HH, Jan JS, Jiang RS, Chao JY, Liang KL, Chen KW, Wu CT, and Lin JC. 2010. Plasma EBV DNA clearance rate as a novel prognostic marker for metastatic/recurrent nasopharyngeal carcinoma. Clin Cancer Res 16:1016-1024. 10.1158/1078-0432.ccr-09-2796

Wong EH, Liew YT, Abu Bakar MZ, Lim EY, and Prepageran N. 2017. A preliminary report on the role of endoscopic endonasal nasopharyngectomy in recurrent rT3 and rT4 nasopharyngeal carcinoma. Eur Arch Otorhinolaryngol 274:275-281. 10.1007/s00405-016-4248-2

You R, Zou X, Hua YJ, Han F, Li L, Zhao C, Hong MH, and Chen MY. 2015. Salvage endoscopic nasopharyngectomy is superior to intensity-modulated radiation therapy for local recurrence of selected T1T3 nasopharyngeal carcinoma - A case-matched comparison. Radiother Oncol 115:399-406. 10.1016/j.radonc.2015.04.024

Yu KH, Leung SF, Tung SY, Zee B, Chua DT, Sze WM, Law SC, Kam MK, Leung TW, Sham JS, Lee AW, Au JS, Hui EP, Sze WK, Cheng AC, Yau TK, Ngan RK, Wong FC, Au GK, and Chan AT. 2005. Survival outcome of patients with nasopharyngeal carcinoma with first local failure: a study by the Hong Kong Nasopharyngeal Carcinoma Study Group. Head Neck 27:397-405. 10.1002/hed.20161

Zou X, Han F, Ma WJ, Deng MQ, Jiang R, Guo L, Liu Q, Mai HQ, Hong MH, and Chen MY. 2015. Salvage endoscopic nasopharyngectomy and intensity-modulated radiotherapy versus conventional radiotherapy in 
treating locally recurrent nasopharyngeal carcinoma. Head Neck 37:1108-1115. 10.1002/hed.23719

408 


\section{Figure 1}

Pre- and post-operative MRI and high-definition endoscopic images

A: Pre-operative MRI shows that the tumor is located in the right pharyngeal recess (rT1). B:

The 6-months post-operative MRI did not show tumor recurrence. C \& D: The 6-months postoperative endoscopic examination images show no visible tumor recurrence. Labels in the pre-operative endoscopic images: a: nasopharyngeal posterior wall, b: right torus tubarius, c: nasopharyngeal carcinoma. Post-operative: a: soft palate, b: nasopharyngeal posterior wall, C: nasopharyngeal right wall. E: Pre-operative MRI shows the tumor invading the left pharyngeal space (rT2). F: Post-operative MRI after 6 months did not show tumor recurrence; G \& H: The 6-months post-operative endoscopic examination images show no visible tumor recurrence. Labels in the Pre-operative endoscopic images: d: nasal septum, e: soft palate, f: right torus tubarius, g: nasopharyngeal carcinoma; Post-operative: $d$ : nasal septum, e: soft palate, f: nasopharyngeal right wall, g: clivus. I: Pre-operative MRI shows the tumor invading the base of the skull (rT3); J: post-operative MRI after 6 months did not show tumor recurrence; $\mathbf{K} \& \mathbf{L}:$ The 6 -months post-operative endoscopic examination images show no visible tumor recurrence. Labels in the pre-operative endoscopic images: h: nasal septum, i: nasopharyngeal posterior wall, j: right torus tubarius, k: nasopharyngeal carcinoma; Postoperative: h: nasal septum, i: nasopharyngeal posterior wall, j: nasopharyngeal right wall, k: clivus. 


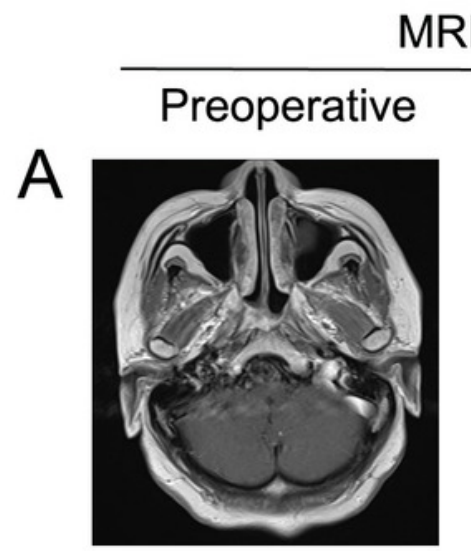

MRI images

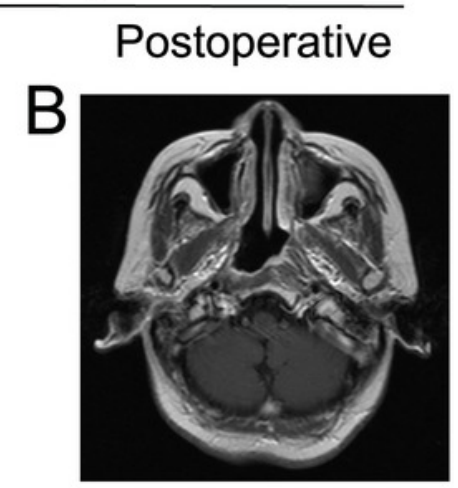

E

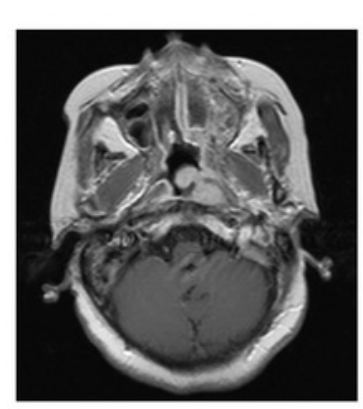

$\mathrm{F}$
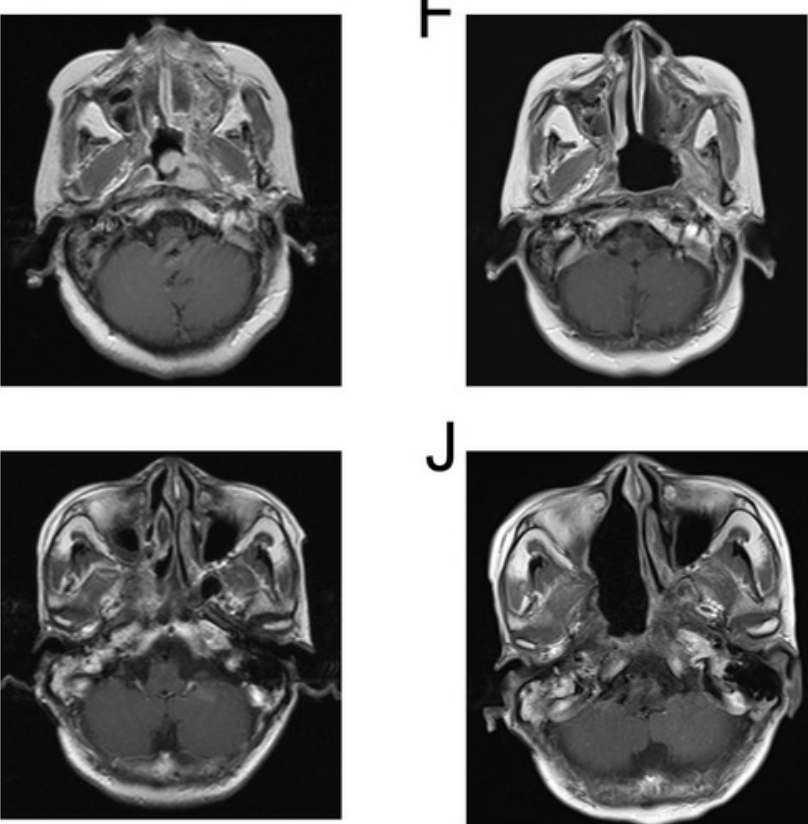

Endoscopic images

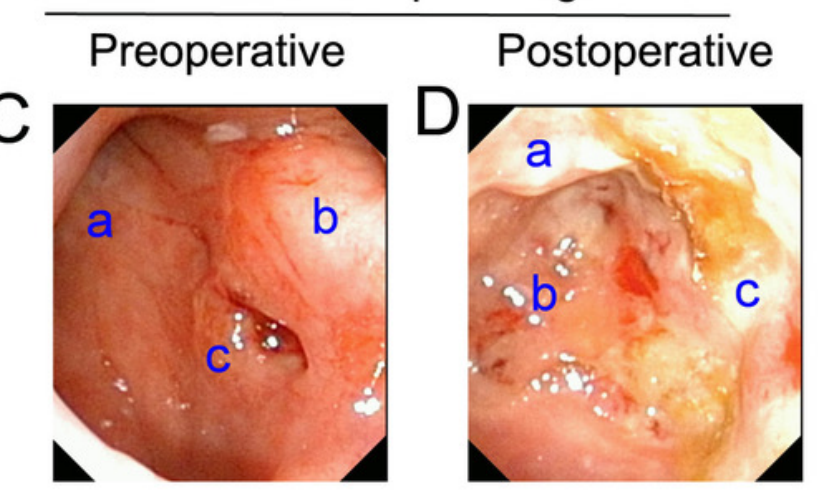

G

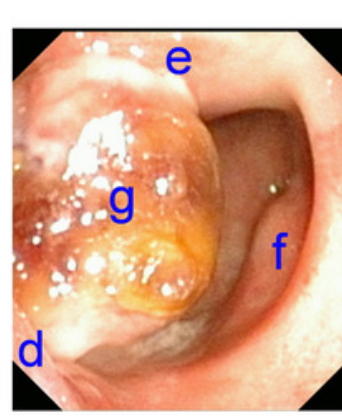

$\mathrm{H}_{\boldsymbol{V}}$

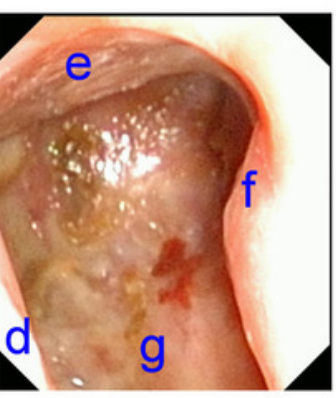

$\mathrm{K}$

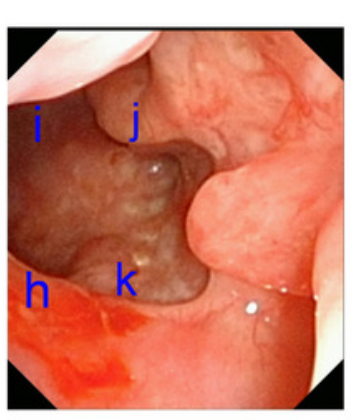

$\mathrm{L}$

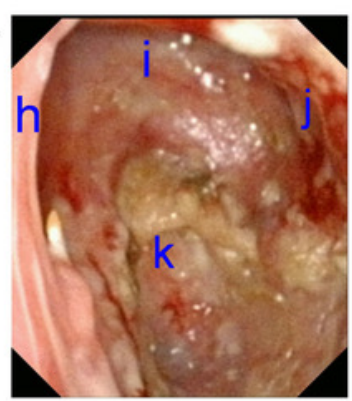


Figure 2

Correlation of EBV-DNA level with staging and treatment methods

A: Comparison of pre-treatment EBV-DNA levels among different T stages; B: Changes in EBV-DNA levels between different treatment methods.

A

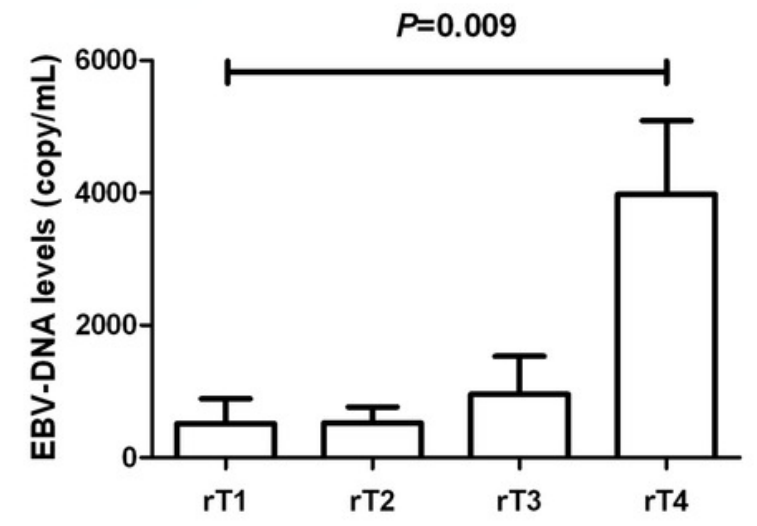

B

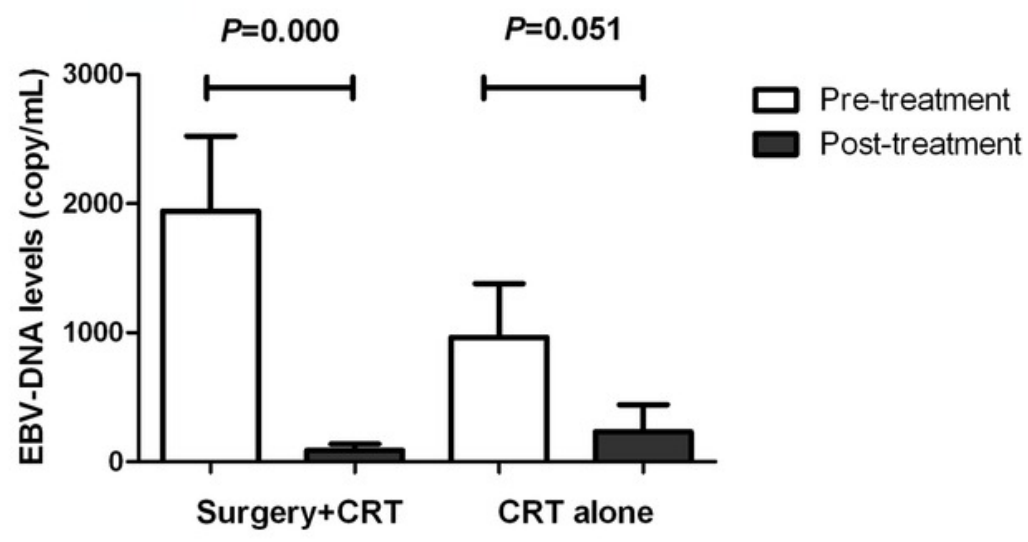


Figure 3

Kaplan-Meier analysis of overall survival among patients with residual or recurrent NPC A: T stages; B: Treatment methods; C: Pre-treatment EBV-DNA; D: Post-treatment EBV-DNA.

A

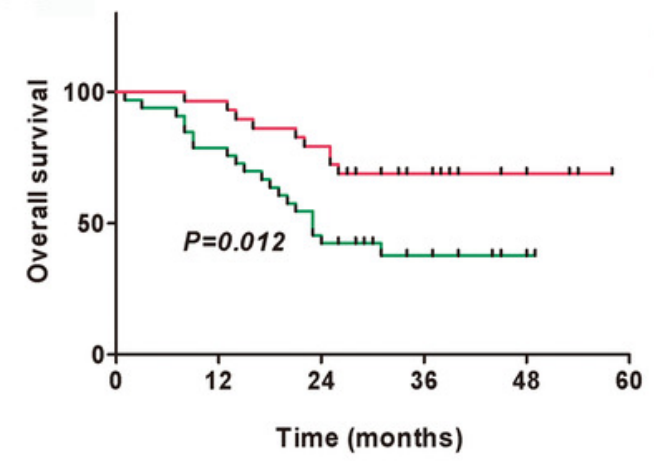

C

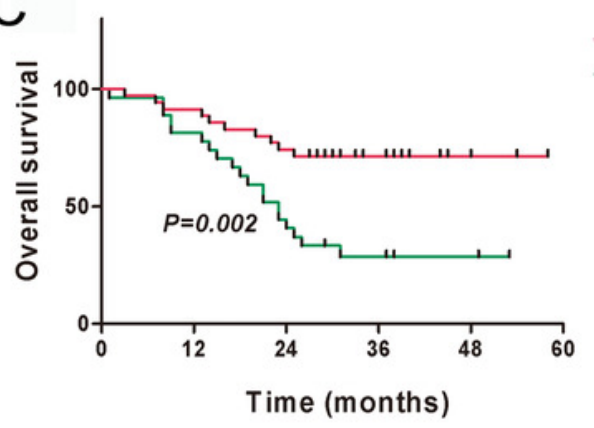

B

$+\mathrm{T} 1+\mathrm{T} 2$

$+\mathrm{T} 3+\mathrm{T} 4$

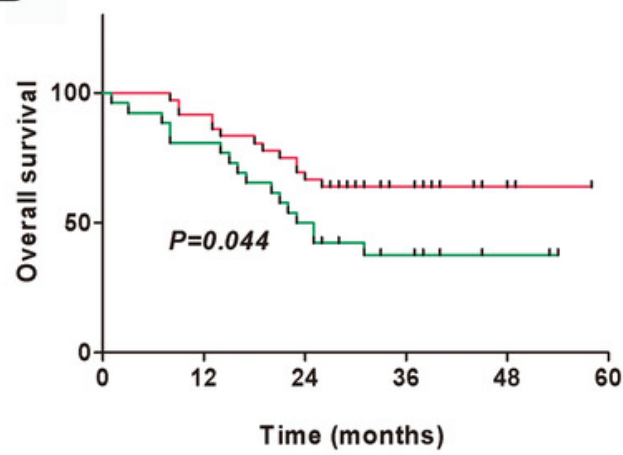

- CRT alone

$\perp$ Surgery + CRT

D

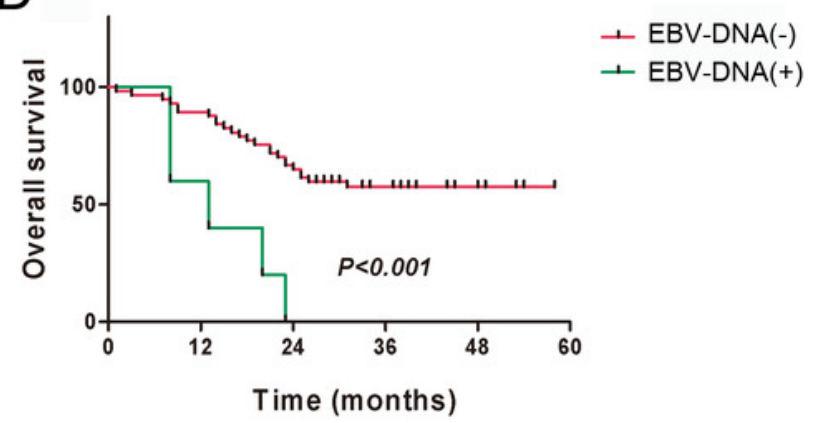




\section{Figure 4}

Kaplan-Meier analysis of disease-free survival among patients with residual or recurrent nasopharyngeal carcinoma

A: T stages; B: Treatment methods; C: Pre-treatment EBV-DNA; D: Post-treatment EBV-DNA.
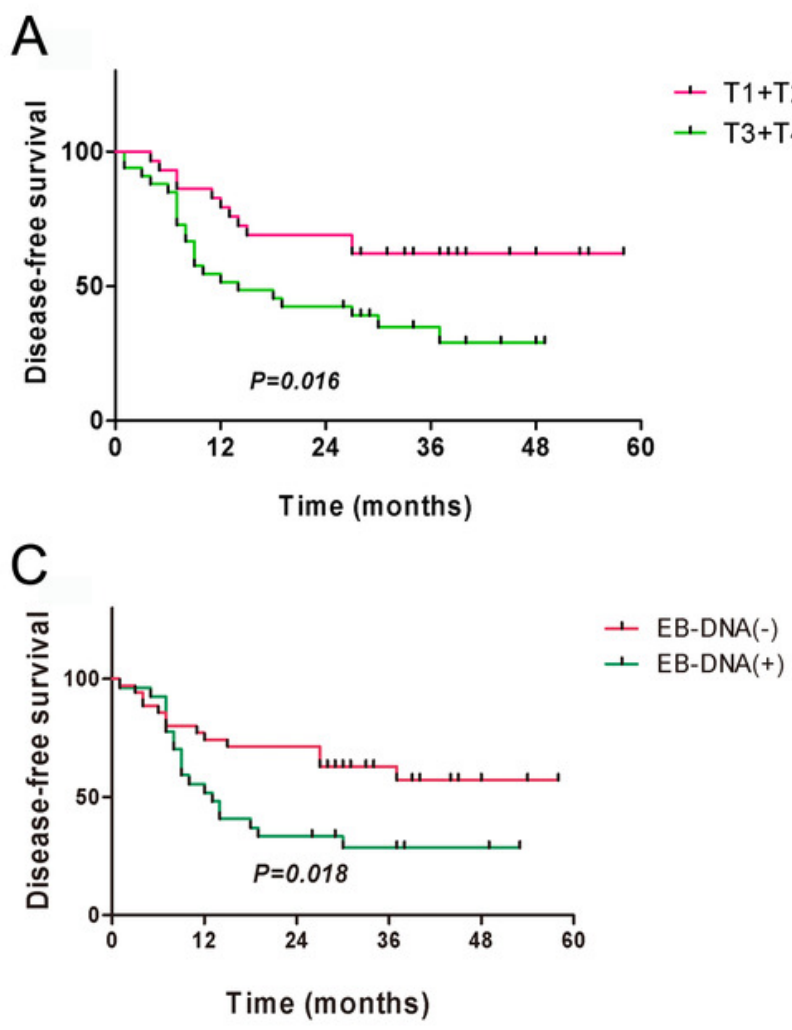

B

$$
\begin{aligned}
& +\mathrm{T} 1+\mathrm{T} 2 \\
& +\mathrm{T} 3+\mathrm{T} 4
\end{aligned}
$$

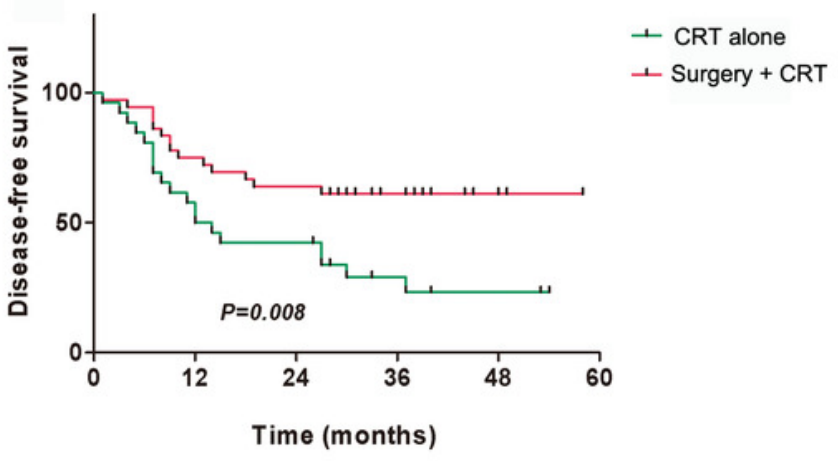

$\mathrm{D}$

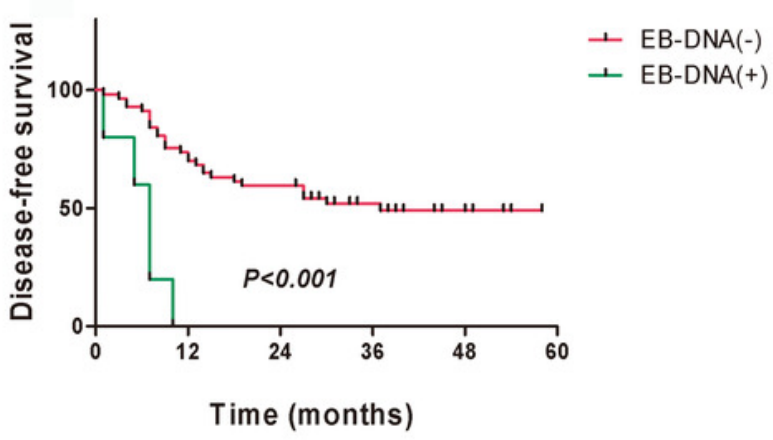




\section{Figure 5}

Kaplan-Meier analysis of clinical prognosis among subgroups of patients with residual or recurrent nasopharyngeal carcinoma

A: Overall survival (OS) comparison of different treatment methods in local early-stage patients; B: Disease-free survival (DFS) comparison of different treatment methods in local early-stage patients; C: OS comparison of different treatment methods in local late-stage patients; D: DFS comparison of different treatment methods in local late-stage patients.

A

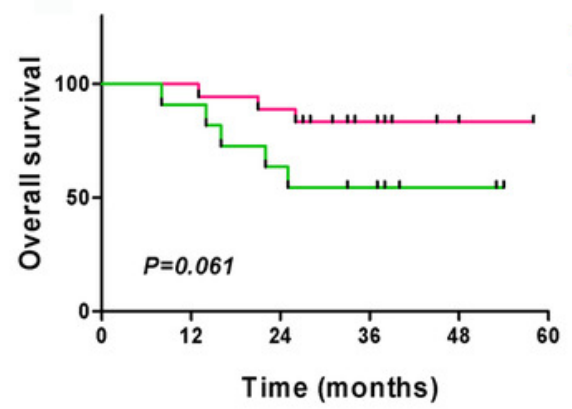

C

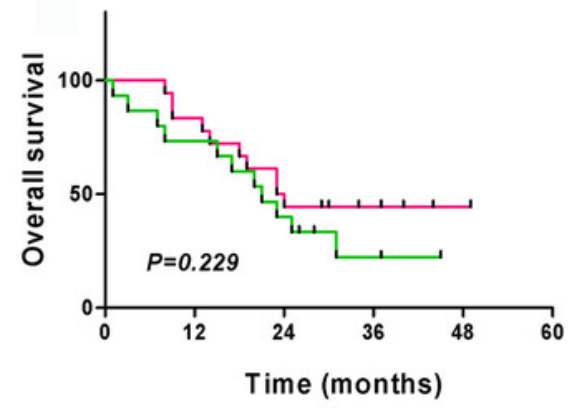

B

+ Early CRT alone

$\perp$ Early Surgery + CRT

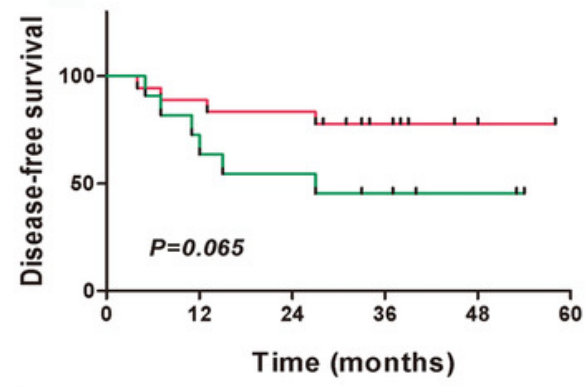

工Early CRT alone

-Early Surgery + CRT

D
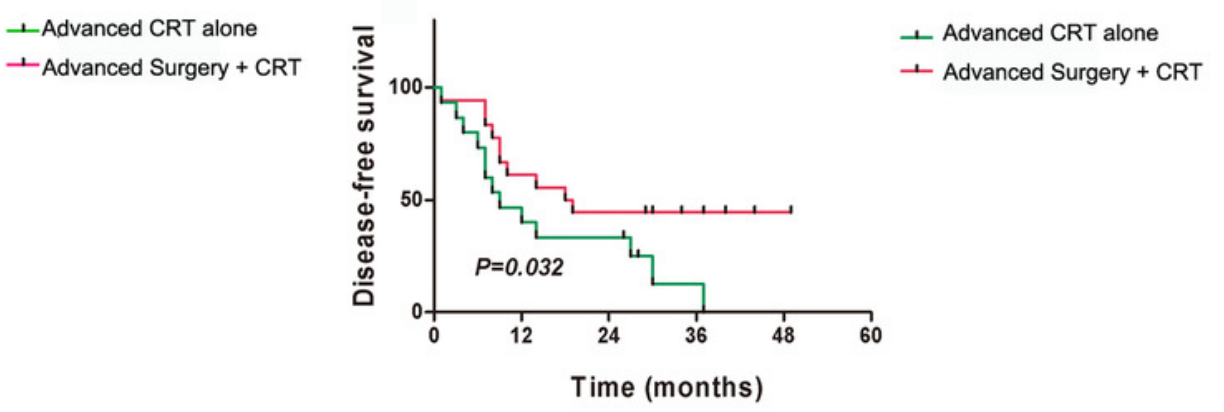
Figure 6

Kaplan-Meier analysis of clinical prognosis among the subgroups of patients with residual or recurrent nasopharyngeal carcinoma

A: Overall survival (OS) of different treatment methods among EBV-DNA(-) patients; B:

Disease-free survival (DFS) comparison of different treatment methods among EBV-DNA(-) patients; C: OS comparison of different treatment methods among EBV-DNA(+) patients; D: DFS comparison of different treatment methods among EBV-DNA (+) patients.

A

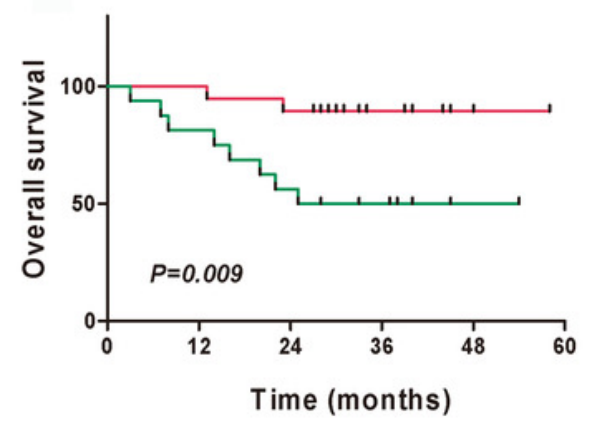

C

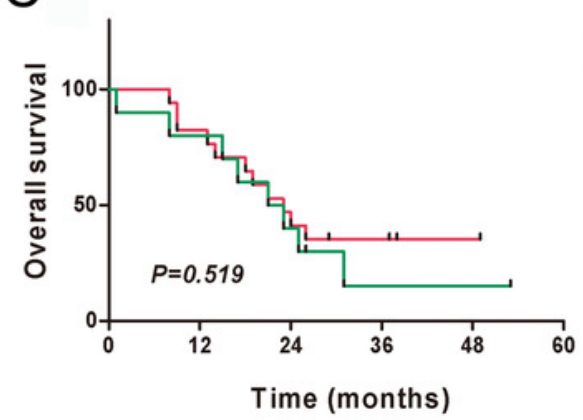

B

-EBV(-) CRT alone

-EBV(-) Surgery + CRT

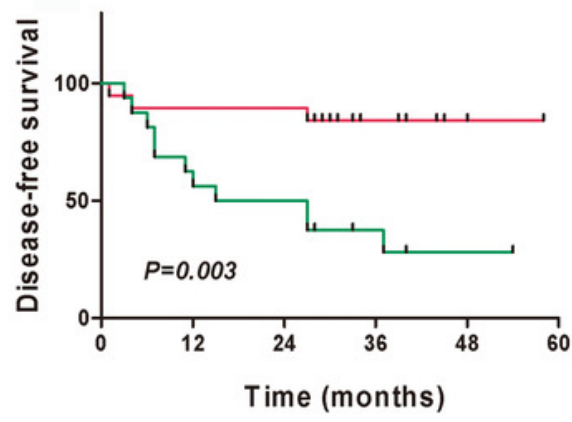

-EBV(-) CRT alone

工EBV(-) Surgery + CRT

D

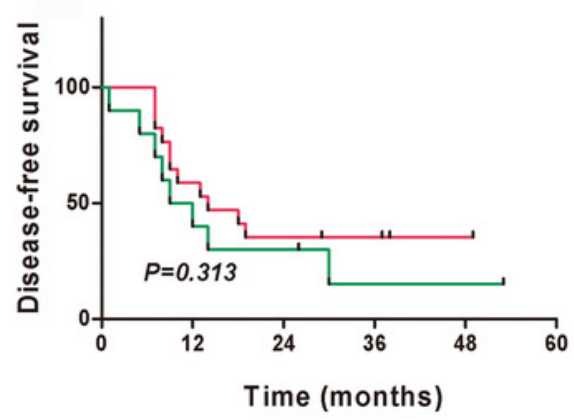

- EBV $(+)$ CRT alone

$-\mathrm{EBV}(+)$ Surgery + CRT 


\section{Table $\mathbf{1}$ (on next page)}

Comparison of clinical data between the surgical group and CRT group

Abbreviations: CRT: chemoradiotherapy. 
1 Table 1. Comparison of clinical data between the surgical group and CRT group

\begin{tabular}{|c|c|c|c|c|}
\hline Factors & Surgical group & CRT group & $\chi^{2}$ & $P$ value \\
\hline \multicolumn{5}{|l|}{ Sex } \\
\hline \multirow[t]{2}{*}{ Male } & 26 & 21 & & \\
\hline & & & 0.601 & 0.438 \\
\hline Female & 10 & 5 & & \\
\hline \multicolumn{5}{|l|}{ Age } \\
\hline \multirow[t]{2}{*}{$<50$ years } & 19 & 12 & & \\
\hline & & & 0.265 & 0.607 \\
\hline$\geq 50$ years & 17 & 14 & & \\
\hline \multicolumn{5}{|l|}{ Local staging } \\
\hline rT1 & 8 & 2 & & \\
\hline \multirow[t]{2}{*}{ rT2 } & 9 & 10 & & \\
\hline & & & 4.465 & 0.215 \\
\hline rT3 & 8 & 9 & & \\
\hline rT4 & 11 & 5 & & \\
\hline \multicolumn{5}{|c|}{ EBV-DNA group } \\
\hline \multirow[t]{2}{*}{ Negative } & 19 & 16 & & \\
\hline & & & 0.471 & 0.492 \\
\hline Positive & 17 & 10 & & \\
\hline
\end{tabular}

2 Abbreviations: CRT: chemoradiotherapy. 
Table 2 (on next page)

Univariate analysis of impact on prognosis

Abbreviations: OS: Overall survival, DFS: Disease-free survival. 
1 Table 2. Univariate analysis of impact on prognosis

\begin{tabular}{lcccc}
\hline & \multicolumn{3}{c}{ OS } & \multicolumn{2}{c}{ DFS } \\
\cline { 2 - 5 } Factor & $\chi^{2}$ value & $P$ value & $\chi^{2}$ value & $P$ value \\
\hline Sex & 0.235 & 0.627 & 0.935 & 0.333 \\
Age group & 0.618 & 0.432 & 1.576 & 0.209 \\
Recurrence time & 0.280 & 0.597 & 0.002 & 0.965 \\
Local staging & 8.954 & 0.030 & 8.194 & 0.042 \\
Treatment method & 4.054 & 0.044 & 7.019 & 0.008 \\
Pre-treatment EBV-DNA & 9.833 & 0.002 & 5.598 & 0.018 \\
Post-treatment EBV-DNA & 13.165 & $<0.001$ & 19.371 & $<0.001$ \\
\hline
\end{tabular}

2 Abbreviations: OS: Overall survival, DFS: Disease-free survival. 


\section{Table 3(on next page)}

Multivariate analysis of impact on prognosis

Abbreviations: OS: Overall survival, DFS: Disease-free survival. 
1 Table 3. Multivariate analysis of impact on prognosis

\begin{tabular}{lcccc}
\hline \multirow{2}{*}{ Factor } & \multicolumn{3}{c}{ OS } & \multicolumn{2}{c}{ DFS } \\
\cline { 2 - 5 } & HR & $P$ value & HR & $P$ value \\
\hline Local staging & 1.515 & 0.043 & 1.685 & 0.004 \\
Treatment method & 0.468 & 0.043 & 0.393 & 0.008 \\
Pre-treatment EBV-DNA & 2.504 & 0.026 & NA & NA \\
\hline
\end{tabular}

2 Abbreviations: OS: Overall survival, DFS: Disease-free survival. 